\title{
Carbohydrates and sulphur-containing compounds in the anaemic buccal epithelium
}

\author{
ALLAN JACOBS \\ From the Department of Pathology, St. Mary's Hospital, London
}

SYNOPSIS Mucosal biopsies from the inside of the cheek show epithelial atrophy, glycogen depletion, and an occasional increase in sulphydryl content in both iron-deficiency and megaloblastic anaemias. These changes are the same in the two types of anaemia but are more marked in the latter group.

Atrophy of the squamous epithelium of the mouth may occur in both iron-deficiency anaemia and anaemia due to vitamin $B_{12}$ deficiency (Jacobs, 1960). The changes are more common and more marked in the latter. They are not related to the degree of anaemia. The occasional finding of hyperkeratotic and parakeratotic lesions in these anaemic mucosae has prompted the investigation of carbohydrates, sulphydryl and disulphide groups, all of which are known to be concerned with the process of keratinization (Rothman, 1954).

\section{MATERIAL}

Suction biopsy specimens were obtained from the buccal mucosa by the method previously described (Jacobs, 1960). The following groups of patients were examined.

1 FORTY-FOUR PATIENTS WITH IRON-DEFICIENCY ANAEMIA All had a haemoglobin concentration of less than $9 \mathrm{~g}$. per $100 \mathrm{ml}$. In only one was the serum iron concentration greater than $60 \mu \mathrm{g}$. per $100 \mathrm{ml}$. and in 21 it was less than $20 \mu$ g. per $100 \mathrm{ml}$.

2 EIGHTEEN PATIENTS WITH MEGALOBLASTIC ANAEMIA DUE TO VITAMIN B $B_{12}$ DEFICIENCY These comprised 16 cases of pernicious anaemia and two cases of multiple jejunal diverticula. Serum $B_{12}$ levels were estimated in 13 of the cases and only in two patients with pernicious anaemia did the level exceed $60 \mu \mu \mathrm{g}$. per $\mathrm{ml}$. In these, values of 100 and $135 \mu \mu \mathrm{g}$. per $\mathrm{ml}$. respectively were obtained.

3 THIRTY-NINE NON-ANAEMIC PATIENTS CONVALESCING FROM OTHER DISORDERS Their ages varied from 19 to 83 years.

\section{METHODS}

All specimens were fixed in $10 \%$ formol saline. They were embedded in paraffin, and $5 \mu$ sections were cut

Received for publication 13 March 1961 vertically through the tissue and stained with haematoxylin and eosin. Periodic-acid-Schiff (P.A.S.) was usedZ to visualize carbohydrate substances (Pearse, 1960). Control sections were treated with salivary diastase at $\frac{\Phi}{3}$ $37^{\circ} \mathrm{C}$. for 30 minutes and some with diastase followed by hyaluronidase (Hyalase) at $37^{\circ} \mathrm{C}$. for 24 hours. Thionin $\stackrel{\mathrm{C}}{-}$ and toluidine blue were both used to investigate meta- $\overrightarrow{0}$ chromasia (Pearse, 1960). Barrnett and Seligman's dihydroxy-dinaphthyl-disulphide (D.D.D.) method as modified by Bahr (1957) was used for the demonstration of sulphydryl groups and its specificity was shown by pre-treatment of control sections with N-ethyl maleimide (Pearse, 1960). Sections treated with thioglycollic acid before incubation with D.D.D. were used to demonstrate total sulphydryl and disulphide concentration (Pearse, $\stackrel{\Omega}{\longrightarrow}$ 1960).

Serum iron was estimated by the method of Ramsay (1957), and serum vitamin $B_{12}$ was kindly estimated by Dr. I. Chanarin using Lactobacillus leishmannii. Marrow? aspirates were fixed in $10 \%$ formol saline and sections stained for haemosiderin using Perls's method (Pearse, 1960).

\section{RESULTS}

EPITHELIAL THICKNESS The maximum epithelial thickness in each specimen is shown in Fig. 1. In the normal group no specimen was found which had a thickness of less than $300 \mu$. In the iron-deficiency group a quarter (11) had a maximum thickness of less than $300 \mu$ and in the group of megaloblastic anaemias over half (10) of the specimens werew thinner than normal. There was no relationship between epithelial thickness and haemoglobin con-o centration, serum iron level, serum vitamin $\mathbf{B}_{12} \Phi$ concentration, or the amount of stainable iron in ${ }^{-}$ the bone marrow in either the iron-deficient or 0 the vitamin- $B_{12}$-deficient patients. Five of the $\frac{\text { Oे }}{\mathbb{D}}$ iron-deficient group showed koilonychia and all@ these had a buccal epithelial thickness of $250 \mu \Omega$ or less. 


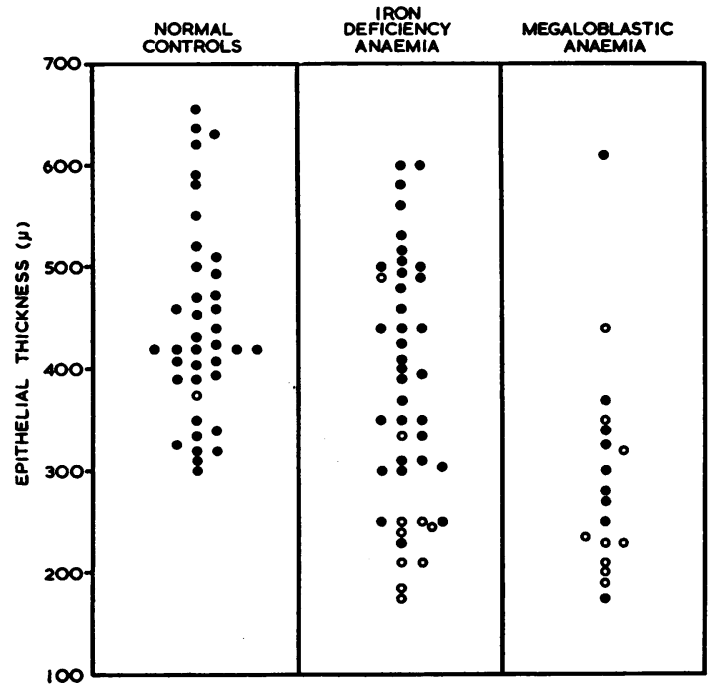

FIG. 1. Maximum epithelial thickness in buccal biopsies from anaemic and non-anaemic patients.

Normal glycogen content

Scanty or absent glycogen

GLYCOGEN Glycogen is demonstrated as a P.A.S.positive substance which is removed by digestion with salivary diastase. The normal buccal epithelium contains abundant glycogen (Fig. 2). It is not present

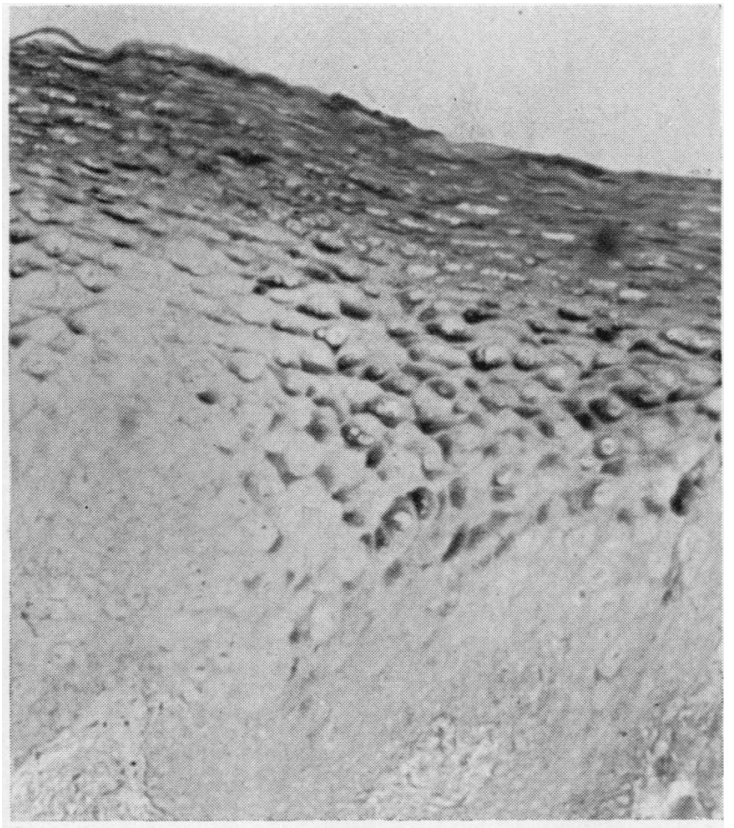

FIG. 2. Normal buccal mucosa. Glycogen present in the superficial part of the epithelium. P.A.S., $\times 150$. in the basal layer but appears in the prickle cell layer and its maximum concentration is just below the surface of the epithelium or sometimes in the surface cells themselves. In some cases the most superficial squamous cells contain no glycogen at all. In one of the non-anaemic group of patients only a trace of glycogen was found in the mucosa.

In 44 cases of iron-deficiency anaemia there were 10 where the epithelium contained only a trace of glycogen or no glycogen at all. Among the 18 cases of megaloblastic anaemia nine showed an absence of glycogen or only trace amounts in the epithelium. In both anaemic groups there is a tendency for the most atrophic epithelium to be deficient in glycogen (Fig. 1). This diminution of glycogen is not apparently related to the level of haemoglobin, serum iron, or serum vitamin $B_{12}$, or to the amount of bone marrow haemosiderin.

NON-GLYCOGEN POLYSACCHARIDES In the normal epithelium a homogeneous substance giving a

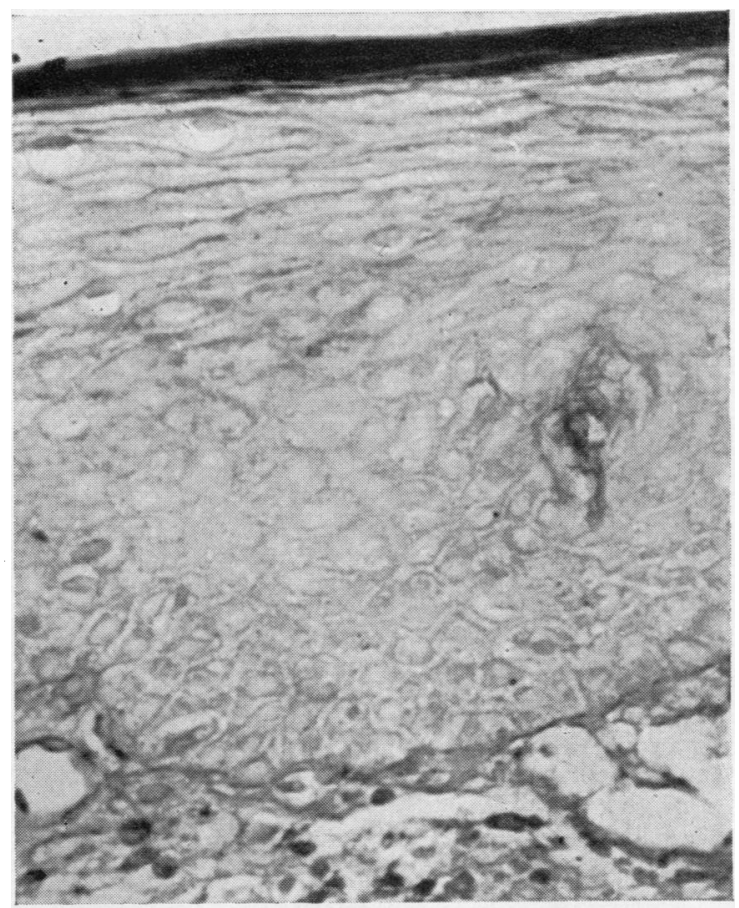

FIG. 3. Atrophic mucosa from a case of iron-deficiency anaemia. Abnormal polysaccharide present in the most superficial cells. P.A.S. following diastase, $\times 300$.

positive P.A.S. reaction and resisting salivary digestion is found in the intercellular spaces. It is particularly prominent and dense in the superficial layers where the intercellular prickles have disappeared and the intercellular space is reduced. 


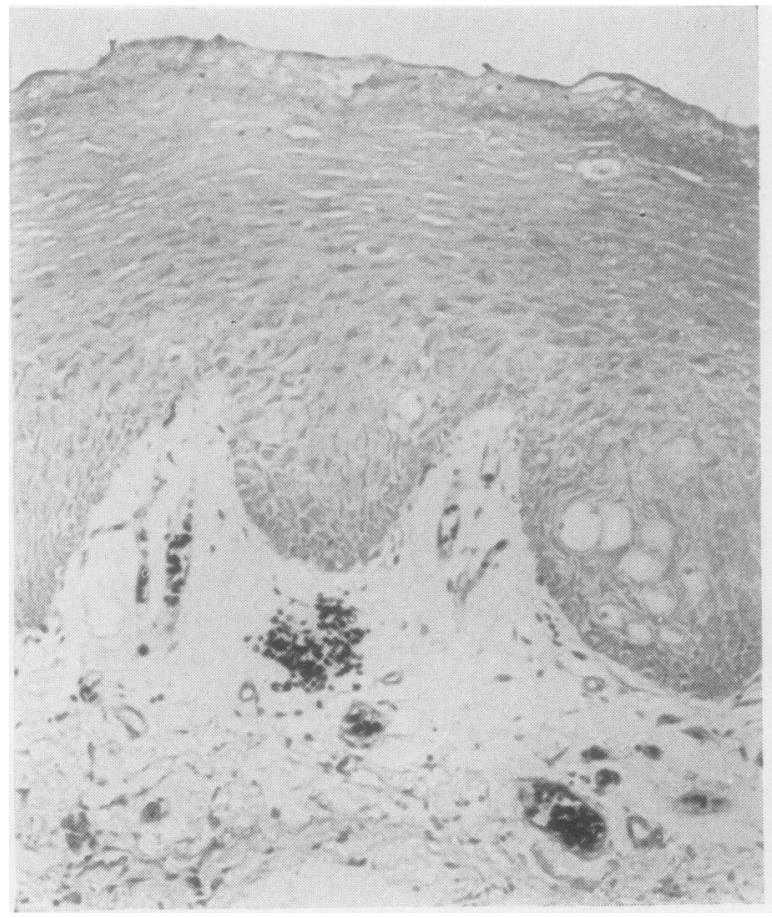

FIG. 4

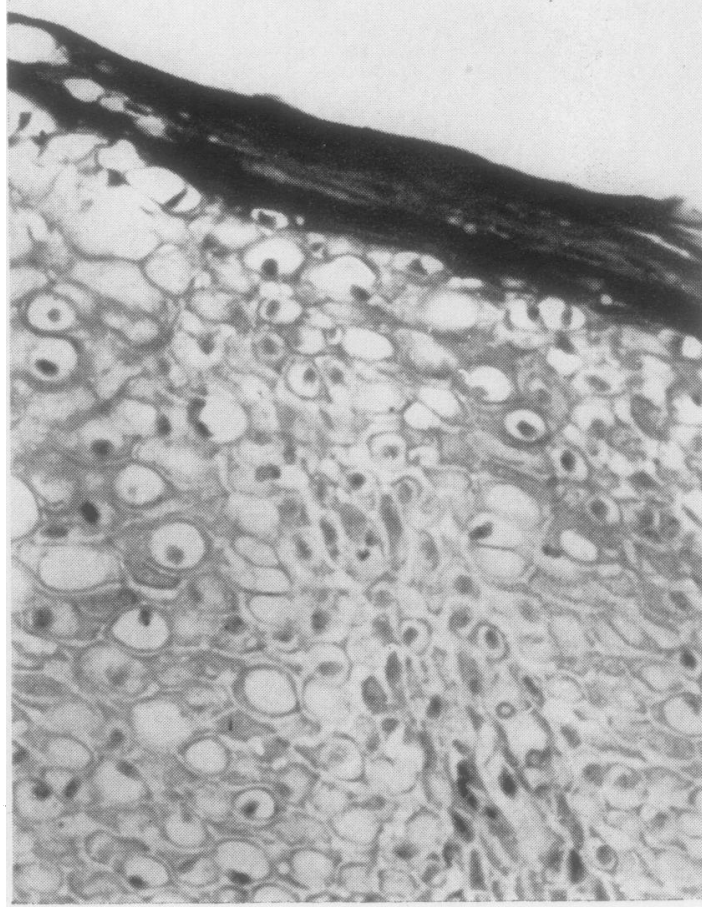

FIG. 5

FIG. 4. Normal buccal mucosa. D.D.D., $\times 150$.

FIG. 5. Atrophic mucosa from a case of iron-deficiency anaemia. There is intense staining in the superficial layer indicating an increase in sulphydryl concentration. D.D.D., $\times 300$.

Staining of the basement membrane by this method varies in intensity between specimens. These polysaccharide substances do not differ in the anaemic and the non-anaemic groups. Very occasional cells in the most superficial layer of the epithelium contain a cytoplasmic fleck of a P.A.S.-positive substance resisting salivary digestion. Only in one normal specimen was more than a trace of this intracellular carbohydrate found. It was present in significant, though still small, amounts in the superficial cells of six cases of iron-deficiency and in four cases of megaloblastic anaemia (Fig. 3). Its presence was not specifically related to the presence or absence of glycogen or to the thickness of the epithelium. It was also unrelated to the degree of anaemia, iron, or vitamin $\mathbf{B}_{12}$ deficiency. In the specimens where this polysaccharide substance was present digestion with hyaluronidase at $37^{\circ} \mathrm{C}$. for 24 hours did not remove it, neither did it show metachromatic staining with thionin or toluidine blue.

SULPHYDRYL AND DISULPHIDE GROUPS All the normal biopsy specimens gave a positive reaction for sulphydryl groups using the D.D.D. method. This was present throughout the full thickness of the epithelium. Staining was considerably reduced by prior incubation with $\mathrm{N}$-ethyl maleimide. In many of the specimens the reaction was weak but in 18 cases there was some increase of staining in the superficial layer (Fig. 4). Six cases showed a slight increase in the amount of staining after treatment with thioglycollic acid.

The appearance in the anaemic groups was similar to the normal except in four cases of iron-deficiency $N$ and in three cases of vitamin $B_{12}$ deficiency which showed an increase in staining, particularly in the superficial layer (Fig. 5). This was entirely due to an increased concentration of sulphydryl groups, as $\sigma$ prior incubation with thioglycollic acid did not $\varrho$ intensify the staining. The increase of sulphydryl $\mathbb{D}$ concentration was invariably associated with an abnormal amount of the intracellular polysaccharide in the superficial cells noted above.

VITAMIN $B_{12}$ In addition to the estimations carried $\stackrel{\mathbb{Q}}{\stackrel{Q}{Q}}$ out in cases of megaloblastic anaemia, serum levels 
were determined in 30 patients with iron-deficiency anaemia, from some of whom biopsies were not taken. All were within the normal range (150 to $850 \mu \mu \mathrm{g}$. per $\mathrm{ml}$.) and showed no relation to the level of haemoglobin, serum iron, or bone marrow haemosiderin.

\section{DISCUSSION}

There have been few studies of the histology of the buccal mucosa and the present findings must necessarily be viewed in the light of observations made on the other squamous epithelial surfaces of the body. Much is known of the epithelium comprising the epidermis and the vaginal lining, though neither of these two sites has precisely the same physiology and pathology as the buccal mucosa. Even within the oral cavity there is probably some variation in the metabolism of the epithelium from one site to another.

Many observations have been made on the normal and abnormal gingiva and the results are of interest. It seems generally agreed that the normal gingival epithelium may contain glycogen (Trott, 1957; Turesky, Glickman, and Fisher, 1959; Weiss, Weinmann, and Meyer, 1959) although Dewar (1955) states that this is only present as a response to inflammation. It is found in about $30 \%$ of cases and Turesky et al. (1959) note that it is usually found in those epithelia which have deep rete pegs. Most of these authors agreed that it is increased in incidence and amount in the presence of gingivitis but Berg, Lundquist, Schram, and Fosdick (1947) found a diminution in inflamed gingiva. There is no variation with age, sex, or menstrual phase and the amount present is not related to the amount of glycogen in the vaginal epithelium (Trott, 1957).

The epithelium covering the alveolar process is probably more akin to the buccal epithelium obtained in the present investigation than the frequently inflamed and traumatized gingival margin. Weiss et al. (1959), comparing the two sites, state that keratinization, which occurs in up to $30 \%$ of gingival specimens, never occurs in the alveolar mucosa. The glycogen of the alveolar squamous cells is also much higher than that found at the gingival margin. In both these respects the picture is similar to that found in the present series of normal buccal epithelia. The alveolar glycogen is apparently unaffected by the presence of inflammation.

Normal skin contains little stainable glycogen (Rothman, 1954) while in the vagina glycogen appears to be inversely related to the degree of keratinization and directly related to the epithelial thickness (Ayre, 1951).

The present series of biopsies confirms the previous observation of epithelial atrophy in some cases of anaemia (Jacobs, 1960) and shows that whereas the normal buccal mucosa usually contains abundant glycogen this is often lacking in anaemic subjects. The glycogen deficiency is usually associated with thinning of the epithelium. It has already been noted that in the gingiva it is the thinner epithelia which tend to be devoid of glycogen. Similarly in the vagina epithelial atrophy following cessation of oestrogen stimulation is associated with a disappearance of glycogen. Although Cruickshank and Sharman (1934) believed that the presence of glycogen in the vagina was the direct result of oestrogen stimulation, Ayre (1951) thought that the presence or absence of glycogen was a direct consequence of the thickness of the epithelium. The accumulation of glycogen in squamous cells some distance from their blood supply, by virtue of their distance from the basement membrane, may be an indication of energy production by an anaerobic mechanism (Ayre, 1951). This view is not entirely supported by the present finding of occasional cases of glycogen depletion occurring in buccal epithelia of normal thickness. Weisberger and Fischer (1960), studying leukoplakia of the cheek, have also noted glycogen depletion in mucosa some distance from the lesions and as yet morphologically normal.

The present results of staining by the D.D.D. method appear to reveal a rather lower concentration of sulphydryl groups in the buccal mucosa than that found in the gingiva by Turesky, Crowley, and Glickman (1957). This may be explained by the occurrence of keratinization in the gingiva. An increase in sulphydryl content was found by these workers in the presence of gingivitis. The increased sulphydryl content in a few of the anaemic mucosae examined may indicate a tendency towards keratinization. These cases always showed, in addition, the presence of an intracellular P.A.S.-positive diastasestable substance in the superficial squamous cells. It is possible that this material may be similar to that found by Steiner (1958) in some parakeratotic lesions of skin. The P.A.S.-positive staining of squamous cell nuclei seen by Steiner was not found in the present series.

The histological findings of epithelial atrophy, glycogen depletion, and a possible tendency to keratinization or parakeratosis in the buccal mucosa appear to be the same in the two types of anaemia investigated. No specific findings characteristic of one group alone were discovered. The only striking difference between the two groups was the occurrence of koilonychia in some of those iron-deficient patients with the severest degree of epithelial atrophy in the mouth. This probably never occurs in megaloblastic anaemias.

Cox, Meynell, Gaddie, and Cooke (1959) 
demonstrated vitamin $B_{12}$ deficiency arising as a consequence of iron-deficiency anaemia. This suggests the possibility that the mucosal changes may all be a result of $B_{12}$ deficiency. The greater prominence of these changes in the megaloblastic group lends support to this possibility. Dabski (1960), basing his conclusions on the observation of exfoliated cells from the mouth, believes this mechanism to operate. Low serum $B_{12}$ levels in iron-deficiency anaemia comparable to those found by Cox et al. (1959) have not been found in the present limited series of cases. Furthermore there is no relation between histological changes and serum vitamin $B_{12}$ levels in either the iron-deficient or megaloblastic cases in this series. The apparently greater effect of the megaloblastic anaemias on the buccal mucosa might be explained by reference to the long period of subclinical $B_{12}$ deficiency which probably precedes all overt clinical signs of pernicious anaemia.

It seems likely that the basic metabolic disorders in the epithelium in iron and vitamin $B_{12}$ deficiency are different. The present investigation, however, has demonstrated only some common manifestations and throws no light on the primary defects.
I should like to thank Professor D. M. Pryce and Mr. I. R. H. Kramer for their encouragement, Miss P. Evans for her technical assistance, and the Medical Research Council for a grant supporting this work.

\section{REFERENCES}

Ayre, W. B. (1951). J. clin. Endocr., 11, 103.

Bahr, G. F. (1957). Acta radiol. (Stockh.), Suppl. 147.

Berg, M., Lundquist, G. R., Schram, W. R., and Fosdick, L. S. (1947). J. dent. Res., 26, 291.

Cox, E. V., Meynell, M. J., Gaddie, R., and Cooke, W. T. (1959). Lancet, 2, 998.

Cruickshank, R., and Sharman, A. (1934). J. Obstet. Gynaec. Brit., Cwlth. 41, 190.

Dabski, H. (1960). Pol. Tyg. lek., 15, 942.

Dewar, M. R. (1955). J. Periodont., 26, 29.

Jacobs, A. (1960). J. clin. Path., 13, 463.

Pearse, A. G. E. (1960). Histochemistry, Theoretical and Applied, 2nd ed. Churchill, London.

Ramsay, W. N. M. (1957), Clin. chim. Acta, 2, 214.

Rothman, S. (1954). Physiology and Biochemistry of the Skin. University of Chicago Press.

Steiner, K. (1958). A.M.A. Arch. Derm., 77, 586.

Trott, J. R. (1957). Aust. dent. J., 2, 283.

Turesky, S., Crowley, J., and Glickman, I. (1957). J. dent. Res., 36, 255.

, Glickman, I., and Fisher, B. (1959). J. Periodont., 30, 116.

Weisberger, D., and Fischer, C. J. (1960). Ann. N.Y. Acad. Sci., 85, Art. 1, p. 349.

Weiss, M. D., Weinmann, J. P., and Meyer, J. (1959). J. Periodont., 30, 209. 